\section{$\underset{\substack{\text { hommes } \\ \text { \& migrations }}}{ }$}

Hommes \& migrations

Revue française de référence sur les dynamiques

migratoires

$1284 \mid 2010$

Migrations et environnement

\title{
Mongolie intérieure. Désertification, migration et transformation des modes de vie
}

\section{Qian Zhang}

Traducteur : Françoise Bouillot

\section{(2) OpenEdition}

\section{Journals}

\section{Édition électronique}

URL : http://journals.openedition.org/hommesmigrations/1239

DOI : 10.4000/hommesmigrations.1239

ISSN : 2262-3353

\section{Éditeur}

Musée national de l'histoire de l'immigration

Édition imprimée

Date de publication : 1 mars 2010

Pagination : 42-55

ISSN : 1142-852X

\section{Référence électronique}

Qian Zhang, " Mongolie intérieure. Désertification, migration et transformation des modes de vie ", Hommes \& migrations [En ligne], 1284 | 2010, mis en ligne le 29 mai 2013, consulté le 30 avril 2019. URL : http://journals.openedition.org/hommesmigrations/1239; DOI : 10.4000/ hommesmigrations.1239 


\section{Mongolie intérieure Désertification, migration et transformation des modes de vie}

Par Qian Zhang, département de Géographie humaine, Université de Stockholm (Suède)

En Mongolie intérieure, le gouvernement chinois a engagé la lutte contre la désertification des steppes et la dégradation des terres. Depuis une dizaine d'années, il encourage la migration et la sédentarisation des éleveurs nomades en milieu urbain. Sur le terrain, si les subventions de l'État poussent certains à partir en laissant derrière eux leurs terres,

d'autres restent profondément attachés à leur mode de vie.

Mais les méthodes traditionnelles de gestion de l'espace, comme la transhumance, sont de plus en plus coûteuses et ne répondent plus aux règles du développement économique. 
La Mongolie intérieure est une vaste steppe, historiquement occupée par différentes tribus pratiquant le pastoralisme et le nomadisme, qui a connu des changements considérables au cours des cent dernières années. L'immigration à grande échelle des paysans Han accompagnée de la réhabilitation des pâturages depuis la fin du XIX siècle et, plus récemment, l'industrialisation et l'urbanisation ont fondamentalement transformé la démographie et le paysage de cette région. Néanmoins, le pastoralisme reste à ce jour une importante ressource pour une forte proportion de la population, notamment les Mongols, et reste le type majoritaire d'usage des terres $^{(1)}$. La Mongolie intérieure, qui fait partie des provinces occidentales, est moins développée que les régions de la côte est de la Chine. Le gouvernement central a cherché à combler cet écart en lançant en l'an 2000 une campagne d'ouverture à l'ouest. La pauvreté rurale vient souvent s'ajouter à la fragilité écologique de certaines régions de Mongolie intérieure. Ces dernières années, les impacts écologiques se sont fait sentir de plus en plus fortement sur fond de changement climatique, avec des conséquences qui touchent autant Beijing et la Chine continentale que la Mongolie intérieure.

\section{Faire face à la dégradation des terres}

La désertification a une longue histoire environnementale dans les régions sèches du nord de la Chine. Bien que la définition et les causes de cette désertification soient aussi contestées que dans les autres parties du monde, elle est largement reconnue par les diverses communautés scientifiques comme l'un des risques environnementaux majeurs en Chine du Nord. On peut dater les premiers projets environnementaux aux alentours de 1978, date du lancement par le gouvernement chinois du Programme de développement sylvicole qui fut appelé plus tard "Forêts des trois Nord", visant à créer une "Grande muraille verte" de 35 millions d'hectares s'étendant sur $700 \mathrm{~km}$ à travers la Chine du Nord. Cependant, la désertification continue à gagner du terrain malgré des tentatives incessantes pour l'endiguer.

La désertification a commencé à susciter de réelles préoccupations quand elle a été associée à la fréquence et à la violence accrues des tempêtes de poussière qui ont frappé Beijing et l'Asie de l'Est depuis la fin des années quatre-vingt-dix. Cette poussière jaune venue des terres arides a beau être un phénomène saisonnier très ancien en Chine, son incidence et son ampleur croissantes en ont fait un point de débat majeur. Elle est devenue aussi un objet de conflit environnemental entre la Chine, le Japon et la Corée du Sud. En outre, elle a posé une menace directe sur les Jeux olympiques de 2008. Les provinces voisines de Beijing, dont la Mongolie intérieure, ont été 
identifiées comme les sources de la poussière et du sable, et de nombreuses ressources ont été consacrées à la maîtrise de la désertification et de la dégradation des terres. Depuis 2002, le gouvernement central s'est engagé à hauteur de 4,7 milliards de yuans (RMB) sur dix ans pour limiter la dégradation des steppes en Mongolie intérieure $^{(2)}$. L'un des discours prévalents sur la désertification en Chine vise les activités humaines. Le programme d'action nationale contre la désertification identifie ainsi ses causes : "Les variations climatiques et les activités humaines, les facteurs humains étant les plus importants ${ }^{(3) "}$. En conséquence, l'une des principales mesures consiste à réduire la pression humaine sur l'usage des terres.

Deux grands programmes ont été mis en ceuvre dans la région rurale de Mongolie intérieure, axés sur deux projets principaux : d'une part, le retour des terres cultivées à la sylviculture, d'autre part l'interdiction des pâtures et la réinstallation des pasteurs nomades - ce qui implique de réinstaller un grand nombre de gens. Les paysans et les pasteurs ont été encouragés, organisés et financés pour qu'ils cessent d'utiliser leurs terres sous contrat et aillent se réinstaller en ville ou dans les zones urbaines. On attend donc que les terres se régénèrent en l'absence d'activités de culture ou de pâture, et que les personnes affectées améliorent leur niveau de vie ailleurs. Il a été prévu de déplacer environ 650000 personnes en Mongolie intérieure entre 2002 et 2008, avec un budget gouvernemental de plus de 100 millions de yuans $(\mathrm{RMB})$.

\section{Objectifs de recherche et zone de l'étude de cas}

Le changement climatique global semble déplacer de plus en plus de gens dans le monde, créant ainsi des "migrants environnementaux". La projection de scénarios futurs exige une meilleure compréhension du rapport entre migration et changement environnemental au niveau local. La migration est "une manifestation et un composant extrêmement complexe de processus économiques, sociaux, culturels, démographiques et politiques également complexes, opérant aux niveaux local, régional,

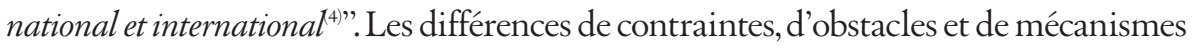
sont purement contextuelles. Cette étude de cas a pour objectif général de découvrir si, et dans quelle mesure, le changement environnemental affecte la décision d'un individu ou d'une famille de migrer.

Le travail de terrain a été mené entre mai et juillet 2008 à Erenhot, une petite ville ayant un hameau de pasteurs sous son administration. Elle a été retenue parce qu'elle fait partie de la ligue Xilin Gol, qui est la préfecture la plus proche au nord de Beijing, 
avec une importante proportion de prairies et une forte population mongole. Dans la ligue Xilin Gol, la réinstallation écologique a été lancée en 2002. Dès 2006, 12859 familles, totalisant 56535 personnes (soit $5,66 \%$ de la population) étaient réinstallées à l'aide de différents programmes, dont 9227 foyers et 41081 personnes par le biais de projets de réimplantation écologique ${ }^{(5)}$. Dans ces derniers projets, 7927 familles, totalisant 37 172 personnes, vivent du pastoralisme $e^{(6)}$.

Erenhot est située sur la fron-

tière avec la Mongolie, dans la partie nord-est de la Région autonome de Mongolie intérieure, sous administration de la ligue Xilin Gol. La ville, qui est un arrêt sur la ligne du Transmongolien, a connu une croissance rapide au cours de ces quinze dernières années, du fait du commerce avec la Mongolie et la Russie. Ce regain d'activité économique accroît la migration et la mobilité, y compris transfrontalière, de la population dans cette zone. La région est située dans une steppe tempérée typique, avec une élévation moyenne de 966 mètres. Geriletuaodu sumu (bourg) est la seule zone pastorale sous l'administration d'Erenhot. Il est composé de quatre gachas (villages) : Erdengobi, Suji, Taoli et Hujiletuya, et d'un village d'élevage laitier, Qiha. La densité de la population est très faible.

\section{Des problèmes environnementaux interconnectés}

Cette zone aride se caractérise par de faibles précipitations, une forte évaporation, une eau rare, un vent fort et des tempêtes de poussière, ainsi qu'une situation écologique fragile. Le premier risque naturel est la sécheresse.

La moyenne des précipitations à Erenhot est de l'ordre de 140 mm, et leur distribution est très inégale tout au long de l'année. Nous voyons sur la figure 2 que les 
précipitations annuelles fluctuent entre trois périodes : 1985 à 1991, 1995 à 1997, 2000 à 2006. Il y a eu des sécheresses extrêmes en 1989, 2001 et 2005. Depuis l'an 2000 , la fluctuation des pluies annuelles s'est encore accentuée. La probabilité d'une sécheresse de printemps est de $60 \%$, celle d'une sécheresse estivale de $53 \%$.

Les tempêtes de poussière surviennent fréquemment au printemps et en automne. La maigre couverture végétale laissée par les sécheresses accroît les effets de la tempête de poussière, qui provoque à son tour un nouveau cycle d'érosion des sols, de dégradation des terres et d'expansion du désert. Après une sécheresse longue avec de fortes températures, il peut aussi survenir des invasions de criquets qui détruisent la végétation des prairies. La plus grave a eu lieu en 2002.

Les épisodes neigeux extrêmes sont rares dans cette région, mais quand ils surviennent, ils provoquent des dégâts considérables. Par exemple, dans l'épisode de neige extrême de 1999 ( $d z u d$ ), beaucoup de familles de pasteurs ont perdu une grande partie de leur cheptel, surtout du gros bétail.

La dégradation des terres est le problème environnemental le plus manifeste dans cette région. Selon les statistiques officielles, 59,96 \% des prairies sont sévèrement dégradées, $29,98 \%$ sont moyennement dégradées, et 10,6 \% sont légèrement dégradées. Ces dernières années, le réchauffement climatique n'a fait qu'empirer ces problèmes interconnectés de sécheresse, de tempêtes de poussière et de dégradation des terres.

La rareté de l'eau et sa qualité médiocre sont aussi un problème dans certaines zones pastorales. En outre, les activités de développement, notamment la construction de routes, l'exploration minière et l'expansion des zones urbaines ont créé de nouvelles causes de conflits sur l'usage des terres.

Figure 2. Précipitations annuelles dans la région d'Jienhot entre 1977 et 2006 


\section{Vers une réinstallation écologique}

Plusieurs chercheurs ont souligné au début des entretiens que la migration environnementale en Chine est fonction de deux types de processus. D'un côté, l'environnement se serait dégradé au point que les gens ne puissent plus survivre à l'endroit où ils se trouvent, de sorte qu'ils sont contraints de partir. De l'autre, le gouvernement, estimant que l'environnement n'offre plus les conditions d'une implantation humaine, organise la réinstallation. Si ces deux mouvements sont imputés à la dégradation environnementale en Chine, leurs mécanismes sont, en pratique, très différents. Le premier type de migration environnementale survient beaucoup moins de nos jours. La dernière grande migration pour raison environnementale est survenue à la fin des années cinquante au cours des "trois années de catastrophes naturelles"(1958-1961).

La migration due à une grave dégradation environnementale survient désormais sur une échelle beaucoup plus réduite dans certaines régions. Par exemple, dans la province de Gansu, où la désertification a empiété sur les terres des paysans, les gens font des allers-retours permanents avec une région pastorale voisine en Mongolie intérieure. Il y a deux raisons à cette baisse des migrations spontanées. D'abord, dans l'histoire chinoise contemporaine, les activités de développement ont laissé peu d'endroits vivables sans occupation humaine. Ensuite, l'économie de planification et le système d'enregistrement ont rendu très difficile toute migration individuelle ou familiale, et à la suite de la réforme économique des années quatre-vingt, la migration rurale-urbaine pour des raisons économiques est devenue le modèle de migration dominant.

\section{Au sujet du volontarisme du gouvernement chinois}

Le gouvernement chinois a commencé à intervenir en 1982. Les gens ont été déplacés hors d'une zone montagneuse dépourvue de ressources au sud de la province de Ningxia où ils pouvaient à peine trouver de quoi vivre dans un environnement extrêmement dégradé. Au début, l'accent a été mis davantage sur l'aide sociale immédiate que sur la protection écologique. Toutefois, ce dernier élément des projets de réinstallation, perçu comme une réponse nécessaire à des risques naturels plus fréquents et plus dangereux, est devenu une priorité depuis 2000. La "réinstallation écologique" est un terme technique pour désigner la réinstallation, organisée par le gouvernement, de gens issus de zones écologiquement fragiles ou de réserves 
naturelles. Les réinstallations écologiques sont essentiellement réalisées dans les régions arides et semi-arides du nord et de l'ouest de la Chine. Ces régions sont très riches en ressources naturelles et les nombreux groupes ethniques qui y vivent en dépendent.

À Erenhot, il y a eu deux projets de réinstallations écologiques, en 2001 et 2006, pour déplacer les pasteurs hors de la zone de pastoralisme. Le premier projet a permis d'organiser les pasteurs pour élever des vaches laitières dans le nouveau hameau de Qiha, et le second les a financés pour qu'ils déménagent dans la ville d'Erenhot. Ces deux contrats étaient d'une durée de cinq ans, au cours de laquelle une famille de pasteurs accepte une interdiction de pâture d'un an sur les prairies qu'elle exploite sous contrat. Depuis 2004, de nombreux pasteurs, qui n'arrivaient plus à joindre les deux bouts dans le village d'élevage laitier, ont choisi de retourner dans leur maison d'origine et ont repris l'élevage du bétail. Le nombre de foyers au village est tombé de 158 à 94, dont 71 élèvent des vaches laitières. Certaines familles sont restées, mais ont repris un berger pour continuer l'élevage de bétail sur leurs pâtures. En outre, seuls 39 foyers de pasteurs sur 94 ont renouvelé leur contrat de réinstallation à l'issue des cinq premières années.

Le second projet de réinstallation a poussé les pasteurs à passer de l'élevage à un travail en ville. Ils se sont vus proposer des maisons, des formations professionnelles, des recherches d'emploi et la possibilité de lancer leur propre entreprise ; en outre, leurs enfants ont été exemptés de tous frais de scolarité et ont bénéficié d'un logement gratuit et de quelques subsides. Un nouveau quartier a été construit pour les pasteurs migrants en 2006.

À ce jour, 180 familles, comptant 580 personnes, ont signé des contrats d'interdiction de pâture à Gerituaodu sumu, et la plupart vivent à Erenhot. Il reste encore 339 foyers de pasteurs, représentant une population de 963 personnes, dont $65,3 \%$ utilisent leurs prairies. Toutefois, d'autres données montrent que seuls 260 foyers élèvent actuellement du bétail.

\section{Des migrations encadrées}

Les destinations de réinstallation se sont modifiées au cours du temps. Dans les années soixante-dix et quatre-vingt, il s'agissait en général d'endroits sous-développés où étaient réinstallés les gens déplacés par la construction de barrages. Dans les années quatre-vingt-dix, certains lieux relativement spacieux, comme des fermes d'État abandonnées, ont été réhabilités pour accueillir des communautés de migrants. À la fin des années quatre-vingt-dix, les emplacements de ce genre sont devenus plus rares, tandis 
qu'un autre processus, l'urbanisation et le développement des petites villes, faisait de ces dernières des destinations de réinstallation. Les autorités ont considéré les petites villes dotées de "cinq équipements" (électricité, eau courante, route, téléphone et service postal) comme une destination idéale pour les migrants écologiques. Les motifs de migrer ou de rejoindre un projet de réinstallation sont assez complexes et varient d'une famille, voire d'un individu à l'autre. Si les problèmes environnementaux ne sont pas en général le motif déterminant dans la décision, ils sont un facteur plus important pour les migrants spontanés que pour les gens déplacés $\odot$ François Gemenne, Each-For par le gouvernement. On ne peut généraliser le schéma selon lequel il s'agirait d'une migration contrainte en ce qui concerne les projets de réinstallation gouvernementaux, et volontaire dans le cas des migrations spontanées, puisqu'il est possible qu'un migrant n'ait pas d'autre option que de déménager, et qu'il soit donc volontaire pour entrer dans un projet de réinstallation gouvernemental.

Pourtant, nous pouvons constater plusieurs différences entre les migrants spontanés et les migrants gouvernementaux. Les premiers tendent à avoir de meilleures capacités de survie et un certain capital social dans leur lieu de destination que les migrants réinstallés de force.

Les résultats de la réinstallation écologique sont très complexes et difficiles à généraliser. Des conflits dans les communautés hôtes ont surgi dans certaines régions et ont souvent été résolus par la coordination des élites. Les migrants doivent affronter de nombreuses difficultés dans leur nouveau site, dont le changement de moyens de subsistance, de style de vie, les problèmes de langue et l'inadaptabilité culturelle. En outre, les nouveaux migrants créent souvent de nouvelles pressions sur l'environnement dans les régions de destination. 


\section{Le rôle des problèmes environnementaux dans la prise de la décision de migrer}

Il est confirmé par la plupart des migrants que très peu de gens ont migré vers une zone urbaine avant le lancement du premier projet de réinstallation. Il semble que la migration constitue bien une option pour gérer la dégradation environnementale dans cette région pastorale. Les pasteurs mongols ont une longue tradition d'équilibrage entre les troupeaux et les pâtures, et de gestion des modèles climatiques extrêmes par la mobilité spatiale. Ils ont un terme particulier pour désigner la migration avec le bétail vers de nouvelles pâtures : "aoter". Bien que les pratiques de pâturage tournant aient été restreintes à des limites de plus en plus étroites, surtout depuis les années quatre-vingt où les pâtures ont été liées par contrat aux foyers et où la plupart des éleveurs se sont stabilisés, on a trouvé, en étudiant les histoires de migration des interviewés, que le pâturage tournant est resté la stratégie la plus souvent utilisée dans les années désastreuses. Dans une certaine mesure, les pasteurs étaient stabilisés, mais pas sédentaires.

Néanmoins, les interviewés indiquent que cette stratégie est de plus en plus opportuniste et coûteuse, et que sa possibilité dépend fortement de leur réseau social. Les flux entre les zones pastorales et rurales sont ainsi devenus un phénomène fréquent. 
Les quinze migrants interrogés pensent que les problèmes environnementaux ont affecté leur décision de déménager à un moment quelconque de leur vie. Onze d'entre eux ont quitté leur foyer d'origine en partie du fait de problèmes environnementaux, ce qui signifie que c'était la raison sous-jacente à leur décision initiale de migrer. Quasiment tous les pasteurs migrants dans cette région ont eu des expériences de migration temporaire avec leurs troupeaux au cours des années de désastre. Toutefois, pour douze d'entre eux, c'est le projet de réinstallation qui a affecté leur décision de partir de chez eux. Quand on a demandé aux non-

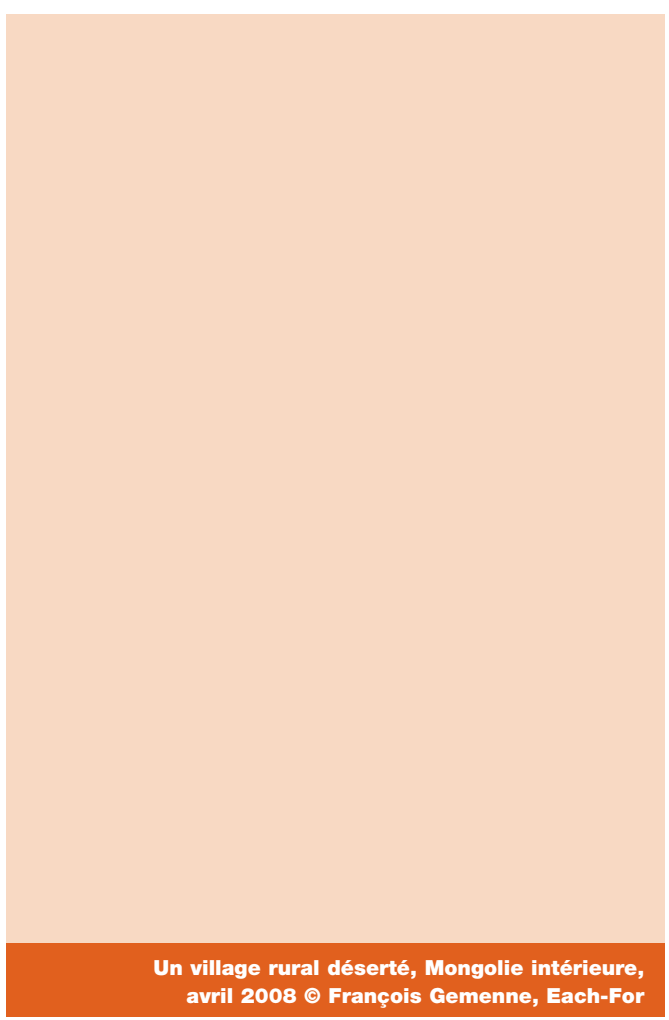
migrants d'exprimer leur opinion sur les migrations des villageois, aucun d'eux n'a donné de réponses positives. Ils ont décrit d'une part les expériences malheureuses des migrants dans le village d'élevage laitier, en soulignant les difficultés à vivre en ville, en soutenant d'autre part que leur meilleur choix était d'être pasteurs. Deux non-migrants ont indiqué que certaines familles ayant une vaste zone de prairies sous contrat ont déménagé à cause de l'importance des subventions offertes. Un autre interviewé a indiqué que ce sont les familles pauvres, possédant peu de têtes de bétail, qui choisissent de partir. Cependant, deux autres disaient aussi qu'il est possible que les jeunes générations préferent rester en ville, et que la tendance future pour elles est peut-être d'y vivre. Les problèmes environnementaux n'étaient pas mentionnés du tout dans leurs réponses.

À l'évidence, la participation volontaire au projet de réinstallation ne signifie pas que l'on pense que la dégradation environnementale est intolérable. Nous soutenons plutôt ici que la réinstallation volontaire est fondée sur une évaluation de facteurs incitatifs. Les problèmes environnementaux sont rarement déterminants dans la décision de migration elle-même, mais ils sont en général sous-jacents à l'action de migration. 


\section{L'attractivité des plans de développement}

Les plans de développement sont reconnus comme étant un important motif de migration par les migrants comme par les non-migrants. Les subventions sont une compensation importante offerte aux migrants. Leur montant dépend de la quantité de prairies sous contrat, et elles sont attribuées au cours de la période de contrat (5 ans renouvelables pour un contrat standard). Elles constituent une motivation pour les familles dont les ressources sont insuffisantes. Cette faiblesse de ressources peut tenir à une pénurie de main-d'cuvre et à la non-récupération de grosses pertes dans les années de catastrophe précédentes. Les familles qui ont migré vers le village d'élevage laitier ont bénéficié de ces soutiens financiers. Dans le premier projet de réinstallation, elles ont toutes reçu des prêts pour acheter des vaches laitières. Le logis et la terre ont aussi été fournis aux migrants. Dans le village laitier, chaque foyer a reçu une maison jumelée et un lopin de terre pour cultiver le fourrage. À Erenhot, on offre des appartements aux migrants. Un meilleur logement doté d'eau courante et d'électricité améliore les conditions de vie, mais peu d'interviewés estiment qu'il s'agit d'un motif important de migration. Les politiques de soutien à la fondation d'entreprise et à l'emploi, notamment une formation gratuite en élevage laitier, créent chez les migrants une forte attente de trouver du travail en ville. Elles sont identifiées comme d'importantes motivations par les interviewés.

Toutefois, plusieurs migrants indiquent qu'ils ont eu de grandes difficultés à obtenir l'emploi stable et pérenne auxquels ils s'attendaient d'après les promesses des politiques. Cette déception a transformé un facteur d'attraction en un facteur de répulsion pour le lieu de destination. 


\section{Les stratégies de survie dans la zone pastorale}

La vie dans la zone pastorale est devenue plus difficile du fait de problèmes environnementaux, sociaux et économiques, mais les individus, les familles et les communautés ont une capacité remarquable à s'adapter à des conditions changeantes et stressantes. Dans la région pastorale d'Erenhot, nous supposons que la migration n'est pas la première option, mais que les gens développent plusieurs stratégies de survie pour gérer les changements climatiques.

L'“aoter", la transhumance, est la première façon d'éviter les risques naturels et de résoudre la pénurie de fourrage, mais elle devient de plus en plus coûteuse et difficile. Certains non-migrants, dont les pâtures d'été ont été dégradées, arrêtent la migration saisonnière entre les pâtures d'été et d'hiver. En revanche, ils choisissent de rester sur les pâtures d'hiver, pourtant sablonneuses et très chaudes en été. D'autres, dans les zones touchées par la pénurie d'eau, n'élèvent pas de gros bétail et transportent souvent l'eau de points éloignés. Mais avec l'augmentation du prix du diesel, le transport est devenu de plus en plus coûteux.

Certains foyers ont commencé à diversifier leurs moyens d'existence. Quelques familles installées à proximité de la ville d'Erenhot se sont lancées dans des 
activités de tourisme, d'autres approvisionnent en produits laitiers des petites boutiques en ville, et certains prennent sous contrat le bétail des migrants pour accroître leurs revenus.

Cependant, cette région souffre d'un manque de coopération entre éleveurs. Il n'y a donc pas d'aide disponible en matière de services financiers, de contributions alimentaires ou d'emplois. Il faut remarquer que tous ces services sont fournis par le système gouvernemental, et que les ONG ou autres groupes similaires ne jouent aucun rôle dans cette région. Il n'y a pas non plus d'organisation interne. Le concept même d'organisation est très étranger aux habitants de cette région pastorale, alors que dans d'autres régions du même type, la coopération entre pasteurs est encouragée.

\section{Conclusion}

Dans cette étude de cas, la dégradation environnementale, le changement climatique notamment et les risques naturels, ont réduit la productivité des prairies et accru la perte de têtes de bétail. Les éleveurs qui ne peuvent plus générer un revenu suffisant sont contraints de migrer. Donc, le motif direct de migration pour raison économique tient en fait indirectement à la dégradation environnementale. Face à cette dernière, la migration est une stratégie de survie pour le gouvernement bien plus que pour les individus. Si la migration peut réduire les stress environnementaux, elle peut aussi augmenter les pressions économiques et sociales dans les nouveaux sites d'installation. En outre, beaucoup d'éleveurs estiment qu'ils ont d'autres moyens de gérer la dégradation environnementale que la migration. Ils pensent qu'affronter les risques fait partie intégrante de la vie pastorale. Leur perception du changement environnemental et de leur identité affecte donc la signification des facteurs environnementaux dans leur décision de migration.

Pour les nombreux migrants qui utilisent les projets de réinstallation, se déplacer est une action temporaire; d'autres, en revanche, ont de fortes attentes d'une vie meilleure dans les destinations offrant des politiques de soutien.

On estime que les problèmes environnementaux dus à l'homme vont s'accroître avec l'accélération de l'urbanisation. Actuellement, les ressources de la zone pastorale sont de plus en plus exploitées au profit du développement urbain. L'empiètement sur les terres, la pollution de l'eau et de l'air et le piétinement des prairies constituent autant de conflits potentiels.

Les éleveurs nomades sont résolument devenus plus vulnérables de nos jours. Mais toute projection des futurs scénarios de migration dans la zone pastorale passe avant 
tout par l'examen des projets gouvernementaux. Certains experts estiment que le gouvernement peut difficilement mener des politiques écologiques aussi "radicales" que celles de 1998, mais au niveau local, les projets de réinstallation écologique actuels vont être maintenus.

\section{Article traduit par Françoise Bouillot}

\section{Bfbliographie}

- N. Bijoor, W. Li, Q. Zhang et G. Huang, "Small-Scale Co-management for the Sustainable Use of Xilingol Biosphere Reserve", Mongolie intérieure, AMBIO: A Journal of the Human Environment, vol. 35, n¹, 2002, pp. 25-29.

- S. Castles et M.-J. Miller, The Age of Migration: International Population Movements in the Modern World, Londres, Macmillan, 1993.

- Y. Chen et H. Tang, "Desertification in North China: Background, Anthropogenic Impacts and Failures in Combating It", in Land Degradation and Development, ${ }^{\circ} 16,2005$, pp. 367-376.

- Chu Chunxia et Meng Huijun, "Problems and Counter-Measures of Environmental Migration in Inner Mongolia", in Northern Economy, 2005, vol. 6, pp. 57-58, (en chinois).

- Amal Kar et Kazuhiko Takeuchi, "Yellow Dust: An Overview of Research and Felt Needs", in Journal of Arid Environments, n59, 2004. pp. 167-187

- Zhigang Liu, \& Yingshun Wang (dir), Meteorological Division in Pastoral Area of Xilingol League Inner Mongolia, Meteorological Publishing House, 2006, (en chinois).

- R. Mearns, "Contextual Factors in the Management of Common Grazing Lands", in Lessons from Mongolia and Northwestern China, 2000. http://www.internationalgrasslands.org/publications/pdfs/tema29_2.pdf

- X. Yang, Z. Ding, X. Fan, Z. Zhou, N. Ma, "Processes and Mechanisms of Desertification in Northern China During the Last 30 Years", in Catena, ${ }^{\circ} 71,2007$, pp. 2-12.

- Qian Zhang, May They Live with Herds-transformation of Mongolian pastoralism in Inner Mongolia of China, thèse de mastère, Université de Trömso, 2006.

Comité national chinois pour la mise en ceuvre de la Convention des Nations unies sur la lutte contre la désertification (CCICCD), Programme national d'action pour combattre la désertification (abstract), 1996. http://www.unccd.int/actionprogrammes/asia/national/2000/china-eng.pdf

\section{Notes}

1. La Région autonome de Mongolie intérieure compte 1715000 personnes vivant dans les zones de pastoralisme, soit $13,6 \%$ de sa population rurale et 7,2 \% de sa population totale (données de 2008). http://www.mof.gov.cn/caizhengbuzhuzhan/zhengwuxinxi/diaochayanjiu/200807/t20080709_56669.html. La Région autonome de Mongolie intérieure compte 68179900 millions d'hectares de prairies naturelles, soit $70,02 \%$ de ses terres agricoles et $59 \%$ de sa superficie totale. http://www.mlr.gov.cn/tdsc/tdgh/200912/t20091222_130487.htm Voir le plan d'occupation des sols de Mongolie, (2002-2010), publié le 22 décembre 2009.

2. N. Bijoor, W. Li, Q. Zhang et G. Huang, "Small-Scale Co-management for the Sustainable Use of Xilingol Biosphere Reserve", Mongolie intérieure, AMBIO: A Journal of the Human Environment, vol. 35, n¹, 2002, p. 30). 3CCICCD, Programme national d'action pour combattre la désertification (abstract), 1996, item 1.3.

4. S. Castles et M.-J. Miller, The Age of Migration: International Population Movements in the Modern World, Londres, Macmillan, 1993.

5. Données issues de Xilingol League Ecological Construction and Rural Population Migration Website. http://wzb.xlgl.gov.cn/44/455/200706/t20070614_20756.htm

6. Données issues de Xilingol League Ecological Construction and Rural Population Migration Website. http://wzb.xlgl.gov.cn/921/986321/200706/t20070615_21941.htm 